\title{
用于细胞和组织中弗林蛋白酶特异性成像的双光子苂光探针研究
}

\author{
刘红文朱隆民娄霄峰袁林张晓兵* \\ (湖南大学化学化工学院 化学/生物传感与计量学国家重点实验室 分子科学与生物医学实验室 长沙 410082)
}

\begin{abstract}
摘要 弗林蛋白酶是前体蛋白转化酶家族中最具特色的酶之一, 具有重要的生物学功能, 其表达量水平与许多疾病有 密切的关系, 如癌症的发生和发展与弗林蛋白酶表达水平有着密切关联. 目前文献中报道了一些单光子荧光探针用于 弗林蛋白酶的检测, 但这些探针不能应用于深层组织成像, 且弗林蛋白酶在肿瘤发展过程的作用仍没有得到很好地研 究. 针对这些问题, 本工作构建了一种新型双光子荧光探针 Nap-F 用于细胞和肿瘤组织内弗林蛋白酶的检测与双光子 成像. Nap-F 是由经典双光子荧光染料 1,8-菜酰亚胺、弗林蛋白酶特异性多肽序列 RVRR 和自消除连接体整合而成. 实 验结果表明 Nap-F 对弗林蛋白酶具有很好的特异性, 能够定量检测弗林蛋白酶的活性. 在飞秒激光 $820 \mathrm{~nm}$ 激发下, Nap-F 能有效降低生物背景, 并提高组织穿透深度, 适用于细胞和组织的双光子成像. Nap-F 成功地实现了几种活细胞 中弗林蛋白酶的双光子成像, 揭示了癌细胞和表达缺陷细胞中弗林蛋白酶含量的差异. 更重要的是, 我们将该探针用 于 $\mathrm{CoCl}_{2}$ 固定 HIF-1 构建的肿瘤细胞缺氧模型成像, 实验结果表明弗林蛋白酶的表达与肿瘤细胞缺氧程度存在正相关 性.
\end{abstract}

关键词 双光子; 苂光探针; 弗林蛋白酶; 癌症; 缺氧

\section{A Two-Photon Fluorescent Probe for Specific Imaging of Furin Activity in Living Cells and Tissues}

\author{
Liu, Hongwen Zhu, Longmin Lou, Xiaofeng Yuan, Lin Zhang, Xiao-Bing* \\ (Molecular Science and Biomedicine Laboratory $(M B L)$, College of Chemistry and Chemical Engineering, \\ State Key Laboratory of Chemo/Biosensing and Chemometrics, Hunan University, Changsha 410082, China)
}

Abstract Furin, the most characteristic member of the proprotein convertase (PCs), has important biological functions. The expression level of furin is related to many diseases, for example, the occurrence and development of cancer is closely related to the expression level of furin. Although several small-molecule fluorescent probes for furin have been developed, which were designed based on near-infrared dye or one-photon dye. These probes exhibit low Stocks' shift or shallow penetration depth, which leading to self-quenching and strong interference. Two-photon fluorescent probes, which utilize two near-infrared photons as the excitation source, can overcome these problems. Herein, a furin-activatable two-photon fluorescent probe (Nap-F) was developed firstly that allowed for detection and imaging of furin in live cells and tumor tissues. Nap-F consists of a classical two-photon fluorophore (1,8-naphthalimide), a furin-particular polypeptide sequence RVRR and a self-eliminating linker. Nap-F is water-soluble and in a fluorescence-off state itself due to the inhibited intramolecular charge transfer (ICT). In the absence of furin, no noticeable fluorescence enhancement was detected, even over 3 days in buffer solution, indicating its good stability. Upon the conversion by furin, it displayed a dramatically fluorescence enhancement at $545 \mathrm{~nm}$, and exhibits high specificity and sensitivity to furin. Nap-F was applied for visualizing the difference in the expression level of furin in various cells, demonstrating its capacity of distinguishing some cancer cells from normal cells. Furthermore, Nap-F was utilized to visualize the variation of furin expression level efficiently after immobilization of hypoxia-inducible factor-1 (HIF-1) by $\mathrm{CoCl}_{2}$, with the results indicating that there is a positive correlation between the expression level of furin and the degree of hypoxia in tumor cells. Owing to the excellent property of Nap-F, the probe was also successful utilized to imaging furin activity in tumor tissues. Thus, Nap-F is able to serve as a potential tool for better exploring the intrinsic link between hypoxic physiological environment and cellular carcinogenesis and detecting cancer in preclinical applications.

Keywords two-photon; fluorescent probe; furin; cancer; hypoxia

\footnotetext{
* E-mail: xbzhang@hnu.edu.cn

Received July 22, 2020; published July 27, 2020.

Supporting information for this article is available free of charge via the Internet at http://sioc-journal.cn.

Project supported by the National Natural Science Foundation of China (Nos. 21890744 and 21877029), National Key R\&D Program of China (No. 2019YFA0210103), and the National Postdoctoral Program for Innovative Talents (No. BX20180093).

项目受国家自然科学基金(Nos. 21890744, 21877029)、国家重点研发计划(No. 2019YFA0210103)和国家博士后创新人才计划(No. BX20180093)资助.
} 


\section{1 引言}

弗林蛋白酶(Furin) 是一种前蛋白转化酶(proproprotein convertases, PCs), 位于反式高尔基体网络 (TGN) 中 ${ }^{[1]}$. 人们对它的结构和功能展开了深入地研究, 发现它对内分泌途径中许多重要的、具有特定基序的前 体蛋白和多肽进行剪切和加工, 使之具有生物学活性以 参与到一系列的生理和病理过程中 ${ }^{[2]}$. 因此, 弗林蛋白 酶展示出重要的生物学功能, 并与许多疾病的发生发展 有着密切的关联. 大量研究表明癌症的发生和发展与弗 林蛋白酶表达水平呈正相关; 与正常细胞相比, 弗林蛋 白酶在卵巢癌、乳腺癌和非小细胞肺癌等癌症中的表达 水平明显增强 ${ }^{[3]}$. 此外, 之前的研究指出, 弗林蛋白酶 的高表达伴随着肿瘤血管的显著减少, 这暗示着弗林蛋 白酶可能与肿瘤缺氧呈正相关 ${ }^{[4]}$. 缺氧是一种常见的肿 瘤发生促进剂, 通常是由于它影响了血管生成和侵袭相 关介质的基因表达, 其中一些介质是弗林蛋白酶的天然 底物. 缺氧促进弗林蛋白酶的表达是通过缺氧诱导因子 -1 (hypoxia-inducible factor-1, HIF-1)的调节, HIF-1 是一 种转录复合物, 在细胞缺氧适应中起着关键作用. 因此, 弗林蛋白酶可以作为除硝基还原酶之外的另一种缺氧 生物标记物 ${ }^{[5]}$. 所以, 对肿瘤细胞中的弗林蛋白酶进行 灵敏、特异地检测和成像, 可以极大地促进肿瘤的诊断 和治疗, 并将推动癌症的发生和转移机制研究, 也有助 于揭示弗林蛋白酶在缺氧中的作用.

苂光成像技术具有灵敏度好、选择性高、空间分辨 率高、实时成像等固有优势, 已在各种生物靶标分析中 得到广泛地应用 ${ }^{[6-10]}$. 苂光探针, 由于具有结构可塑性 和信号输出可调性, 是荧光成像分析各种生物靶标的有 力工具. 其中, 小分子荧光酶底物探针是实时成像活细 胞中酶活性的理想工具 ${ }^{[11]}$. 目前只有几种基于近红外 染料或单光子染料的小分子荧光探针用于弗林蛋白酶 的检测和成像 ${ }^{[12-16]}$. 然而, 这些探针的斯托克斯位移较 小, 容易导致荧光自猝灭和瑞丽散射干扰; 或激发波长 较短, 导致组织穿透深度较浅. 双光子(two-photon)荧光 探针以两个近红外光子作为激发光源, 因而生物背景荧 光低、组织穿透深度深, 已被广泛应用于各种生物靶标 的检测和成像 ${ }^{[17-21]}$. 因此, 开发新型的双光子苂光探针 用于双光子成像活细胞和组织中的弗林蛋白酶活性具 有重要意义和挑战.

在本工作中, 我们首次构建了一种弗林蛋白酶激活 的双光子荧光探针 (Nap-F), 并用于细胞和肿瘤组织中 的弗林蛋白酶活性选择性地成像. 我们选取经典的双光 子染料 1,8 -芸酰亚胺作为荧光团, 再将弗林蛋白酶特异 性肽链序列 RVRR 通过连接体与苂光团整合得到探针 Nap-F(图 1). 探针 Nap-F 是基于分子内电荷转移(ICT) 机理设计的, 荧光团的 C-4 酚羟基与连接体以氨基甲酸 酯连接起来, 使得 C-4 酚羟基的供电子能力显著减弱, ICT 过程被抑制, 探针本身在飞秒激光 $820 \mathrm{~nm}$ 激发或单
光子 $420 \mathrm{~nm}$ 激发下, 在 $545 \mathrm{~nm}$ 处的荧光均很弱. 而探 针 Nap-F 在被弗林蛋白酶剪切后, 迅速发生分子内自消 除反应, 进而释放出苂光团, 得以实现单双光子激发下 弗林蛋白酶的 “增强” 型检测. 得益于探针结构上的三 个胍基, 探针具有很好的水溶性; 此外, 由于连接体的 结构特性, 探针具有很好的稳定性, 在不含弗林蛋白酶 的检测体系中, 能够稳定存放至少 $3 \mathrm{~d}$. 探针经弗林蛋 白酶转化后, 在 $545 \mathrm{~nm}$ 处显示出约 25 倍荧光增强, 且 对弗林蛋白酶具有很好的特异性和灵敏度. 我们将探针 Nap-F 用于成像不同细胞间弗林蛋白酶表达的差异性, 显示探针具有区分癌细胞和正常细胞的能力. 此外, 基 于缺氧条件下 HIF-1 与弗林蛋白酶含量之间密切相关 性, 我们利用 Nap-F 成功地观察到 $\mathrm{CoCl}_{2}$ 固定 HIF-1 后 弗林蛋白酶表达水平的变化, 并进一步探讨缺氧生理环 境与细胞癌变的关系. 最后, 由于 Nap-F 的优良双光子 光学性质, 该探针也成功地用于肿瘤组织中弗林蛋白酶 活性的成像.

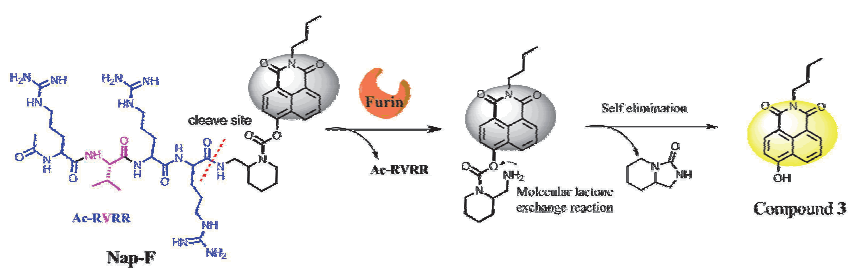

图 1 探针 Nap-F 的结构和其响应机理示意图

Figure 1 The response mechanism of Nap-F to furin

\section{2 结果与讨论}

\section{1 探针的设计与合成}

近年来，一些荧光探针被报道并证实了弗林蛋白酶 在生理和病理过程中的重要作用. 然而, 以往的探针大 多采用单光子染料设计, 限制了其在深部组织成像中的 应用. 因此, 我们选用 4-差基-1,8-荎酰亚胺为双光子荧 光团, 以肽序列 Ac-Arg-Val-Arg(Ac-RVRR)为弗林蛋白 酶特异性切割底物 ${ }^{[22,23]}$, 构建探针 Nap-F. Nap-F 中的自 消除连接物(2-氨甲基哌啶)不仅可以减少酶识别的空间 位阻, 而且可以稳定探针的结构, 以减少探针的自水解, 消除假阳性信号 ${ }^{[24-26]}$. 用不同的肽序列(Ac-RRRV)合成 了不能被弗林蛋白酶剪切的对照探针 Nap-C(图 S1), 以 证实 Nap-F 对弗林蛋白酶的特异性. 探针 Nap-F 和 Nap-C 的合成如图 S2 所示, 新化合物的结构通过核磁 氢谱 ( ${ }^{1} \mathrm{H}$ NMR) 和质谱(MS)进行了表征.

\section{2 体外响应以及选择性测试}

以酶解法裂解约 $2 \times 10^{8}$ 个 MDA-MB-468 细胞, 得 到的细胞裂解液作为弗林蛋白酶的来源. 如图 $2 \mathrm{~A}$ 所示, 在不加入细胞裂解液的情况下, 以 $420 \mathrm{~nm}$ 激发 Nap-F 和 Nap-C 的缓冲溶液, 都只检测到微弱的荧光发射; 而 加入细胞裂解液后, 细胞裂解液中的弗林蛋白酶激活探 针 Nap-F，溶液的苂光强度明显增强，而 Nap-C 溶液中 
的苂光强度没有明显变化. 且溶液的荧光强度随时间增 长逐渐增强, 在 $5 \mathrm{~h}$ 内达到饱和 (图 2B); 此外, 如图 2C 所示探针 Nap-F 与不同体积的细胞裂解液在 $37^{\circ} \mathrm{C}$ 下捊育 $5 \mathrm{~h}$ 后, 溶液的菼光增强倍数与细胞裂解液的体积成正相 关性, 这表明 Nap-F 具有定量分析弗林蛋白酶活性的能 力 (图 2C). 且前 $90 \mathrm{~min}$ 内, 体系的荧光增强了近 10 倍, 与之前报道的文献相比, 探针 Nap-F 展现出更快的响应 速度. 我们还研究了 Nap-F 在细胞培养基 (DMEM) 中 的稳定性, 结果是探针即使在细胞培养基中放置 $3 \mathrm{~d}$ 也没 有检测到明显的苂光增强，而在加入细胞裂解液后，检 测到明显的信号增强(图 2D). 这说明 Nap-F 在细胞培养 基中非常稳定, 不会发生自水解以产生假阳性信号. 综 上结果表明探针 Nap-F 适用于缓冲溶液中弗林蛋白酶的 检测, 在活细胞中有进一步的应用前景.

接下来, 我们考察了 Nap-F对弗林蛋白酶的选择性, 以确定 Nap-F 是否适用于复杂的生物体系. 如图 S3 所 示, 只有 MDA-MB-468 细胞裂解液的加入引起显著的 荧光增强. 这表明 Nap-F 对弗林蛋白酶具有高度的特异 性, 这是因为探针骨架中的特异性底物序列 (Ac-RVRR). 探针 Nap-F 展现出良好的选择性, 有助于 探索弗林蛋白酶在生物和生理过程中的作用. 我们将探 针和细胞裂解液反应产物简单纯化后, 质谱结果表明反 应产物确是化合物 $\mathbf{3}$, HPLC 结果也证明了探针的反应机 理 (图 S4, S5).
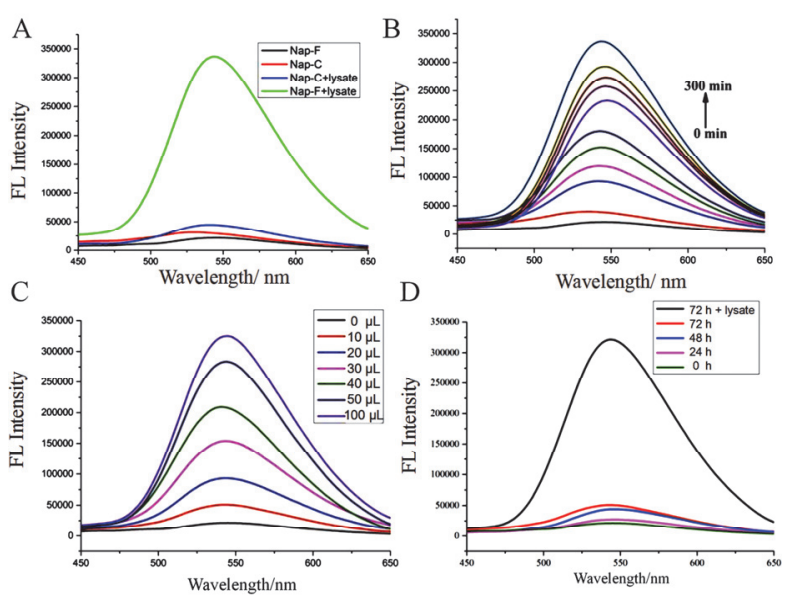

图 2 Nap-F 的苂光响应曲线. (A) Nap-F 和 Nap-C 在 MDA-MB-468 细胞裂解液存在或不存在时的苂光响应光谱; (B) Nap-F 的时间响应 曲线; (C) Nap-F 与不同体积的 MDA-MB-468 细胞裂解液的荧光响应 曲线; (D) Nap-F 在 PBS 体系中放置 1, 2, $3 \mathrm{~d}$, 及与 $100 \mu \mathrm{L}$ 细胞裂解液 反应 $5 \mathrm{~h}$ 后荧光光谱. $\lambda_{\mathrm{ex}}: 420 \mathrm{~nm}, \lambda_{\mathrm{em}}: 450 \sim 650 \mathrm{~nm}$.

Figure 2 (A) Emission spectrum of Nap-F $(5 \mu \mathrm{mol} / \mathrm{L})$ and Nap-C (5 $\mu \mu \mathrm{mol} / \mathrm{L}$ ) in the absence/presence of MDA-MB-468 cell lysate; (B) The time-course response of Nap-F after incubation with $100 \mu \mathrm{L}$ cell lysate for $0 \sim 300 \mathrm{~min}$; (C) Nap-F in the presence of various volumes of above cell lysate. (D) The fluorescence intensity of Nap-F after incubation in PBS for $0 \sim 3 \mathrm{~d}$ or after incubation with $100 \mu \mathrm{L}$ cell lysate for $5 \mathrm{~h}$. $\lambda_{\text {ex: }}$ : $420 \mathrm{~nm}, \lambda_{\mathrm{em}}: 450 \sim 650 \mathrm{~nm}$.

\section{3 活细胞荧光成像}

首先, 我们用标准 MTS 法测试了探针 Nap-F 对
MDA-MB-468 细胞活性的影响. 如图 S6 所示，在 0 20 $\mu \mathrm{mol} / \mathrm{L}$ 的探针浓度范围内, 探针 Nap-F 对培养细胞的细 胞毒性较低, 说明 Nap-F 适合于生物成像. 鉴于其在缓 冲溶液中展现出来的良好性能, 我们接下来将 Nap-F 应 用于活细胞中弗林蛋白酶活性的苂光成像. HeLa、

HepG2、MDA-MB-468 和 Lovo 细胞分别与探针 Nap-F (5 $\mu \mathrm{mol} / \mathrm{L}$ ) 在 $37{ }^{\circ} \mathrm{C}$ 下卯育 $30 \mathrm{~min}$, 用 DPBS 洗涤后再进行 苂光成像. 如图 3A 和图 S7 所示, 在 HeLa、HepG2 和 MDA-MB-468 细胞中观察到明亮的苂光信号, 但 Lovo 细胞中荧光信号很弱, 这是因为 HeLa、HepG2 和 MDA-MB-468 细胞系过度表达弗林蛋白酶, 而 Lovo 细 胞是一种弗林蛋白酶缺陷的人类结肠腺癌细胞 ${ }^{[15,23]}$. 在 另一组对照实验中, MDA-MB-468 细胞与对照探针 Nap-C 共孵育, 荧光成像结果显示在 MDA-MB-468 细 胞中检测不到苂光信号 (图 3B). 结果表明, 在单光子 和双光子显微镜下, 探针 Nap-F 能够选择性检测活体细 胞内弗林蛋白酶活性并成像.

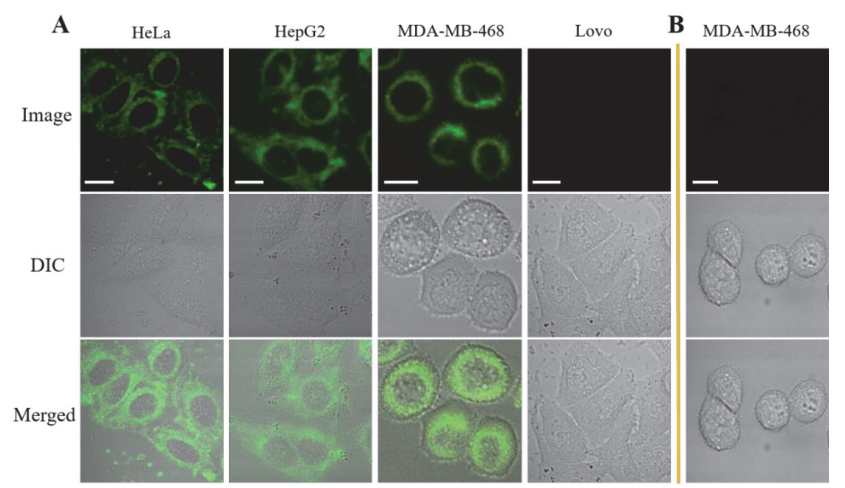

图 3 (A) Nap-F $(5 \mu \mathrm{mol} / \mathrm{L})$ 在不同细胞中的双光子荧光成像; (B) Nap-C 在 MDA-MB-468 细胞中的双光子荧光成像; $\lambda_{\mathrm{ex}}: 820 \mathrm{~nm}, \lambda_{\mathrm{em}}$ : $450 \sim 600 \mathrm{~nm}$, 比例尺: $20 \mu \mathrm{m}$.

Figure 3 (A) Two-photon fluorescent imaging of HeLa, HepG2, MDA-MB-468 cells after being incubated with Nap-F $(5 \mu \mathrm{mol} / \mathrm{L})$ for 30 min ; (B) Two-photon fluorescent imaging of MDA-MB-468 cells after being incubated with Nap-C $(5 \mu \mathrm{mol} / \mathrm{L})$ for $30 \mathrm{~min}$. $\lambda_{\mathrm{ex}}: 820 \mathrm{~nm}, \lambda_{\mathrm{em}}$ : $450 \sim 600 \mathrm{~nm}$, scale bar: $20 \mu \mathrm{m}$.

缺氧是实体肿瘤的共同特征，缺氧环境通过 HIF-1 的固定化，极大地促进血管的生成. 在正常氧气浓度条 件下，HIF 经脯氨酰着化酶羟基化后，被肿瘤抑制蛋白 识别并泛素化, 导致其被降解; 但在缺氧条件下, 这一 过程被抑制, HIF 在细胞核中持续积累，起转录因子的 作用，反过来促进多种促血管生成因子的表达，如血管 内皮生长因子(VEGFs)、血管生成素(ANGPTs)、血小板 衍生生长因子(PDGFBs), 以促进新血管的形成. 这些涉 及血管生成过程的各种促血管生成因子是不成熟的前 体蛋白, 需要通过弗林蛋白酶等前体蛋白转换酶的作用 将他们转变为成熟的形式, 以发挥其功能. 我们以 $\mathrm{CoCl}_{2}$ 诱导细胞内 HIF-1 的固定化, 以构建细胞缺氧的 生理环境 ${ }^{[27]}$. 如图 $4 \mathrm{~A}, \mathrm{~B}$ 所示, 用 $100 \mu \mathrm{mol} / \mathrm{L} \mathrm{CoCl}_{2}$ 对 HepG2 细胞进行预处理, 随着预时间 $(0 \sim 48 \mathrm{~h})$ 的延 
长, 细胞内苂光信号逐渐增强. 此外, 在另一组实验中, 我们采用不同浓度的 $\mathrm{CoCl}_{2}(0 \sim 100 \mu \mathrm{mol} / \mathrm{L})$ 对细胞进 行预处理 $48 \mathrm{~h}$. 结果显示, 随着 $\mathrm{CoCl}_{2}$ 浓度的增加, 细胞 中的荧光信号逐渐增强(图 $4 \mathrm{C}, \mathrm{D}$ ). 因此, 我们可以得 出结论: 随着细胞缺氧程度的加剧, 细胞内弗林蛋白酶 的活性逐渐升高, 从而更多的染料被释放出来, 表现为 荧光信号的逐渐增强. 因此, 探针 Nap-F 能够用于肿瘤 细胞缺氧检测. 目前大多数缺氧荧光探针是基于硝基还 原酶等设计的, 而这类探针容易受到肿瘤细胞还原性物 质, 如 GSH, 和细菌感染的干扰, 而探针 Nap-F 能够有 效克服这些问题, 或许能够成为一种高保真的肿瘤缺氧 探针.

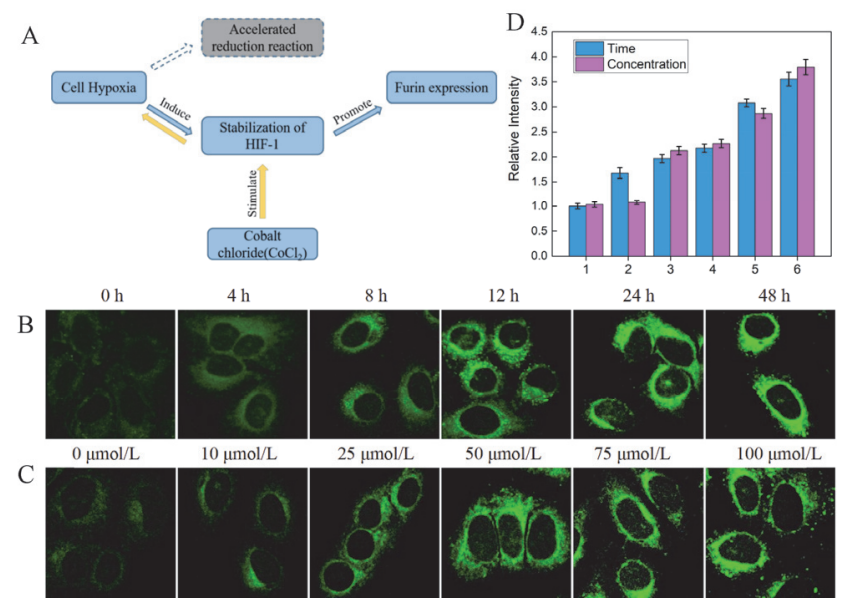

图 4 (A) 细胞缺氧、HIF-1 与弗林蛋白酶的内在联系示意图; HepG2 细胞缺氧成像, 细胞经 $\mathrm{CoCl}_{2}$ 预处理后, 与探针 Nap-F $(2 \mu \mathrm{mol} / \mathrm{L})$ 共 餒育 $30 \mathrm{~min}$ 后成像, (B) 保持 $\mathrm{CoCl}_{2}$ 浓度为 $100 \mu \mathrm{mol} / \mathrm{L}$, 诱导 $\mathrm{HIF}-1$ 固定化不同时间后 $(0 \sim 48 \mathrm{~h})$ 的细胞成像; (C) 采用不同浓度 $\mathrm{CoCl}_{2}$ $(0 \sim 100 \mu \mathrm{mol} / \mathrm{L})$ 诱导 HIF-1 固定化 $48 \mathrm{~h}$ 后的细胞成像; (D) 图 $4 \mathrm{~B}, \mathrm{C}$ 各图的平均苂光强度, 将 $0 \mathrm{~h}$ 或者 $0 \mu \mathrm{mol} / \mathrm{L}$ 的图的苂光强度定义为 1.0. $\lambda_{\mathrm{ex}}: 820 \mathrm{~nm}, \lambda_{\mathrm{em}}: 450-600 \mathrm{~nm}$.

Figure 4 (A) Intrinsic Relationship between Hypoxia, Furin, and Tumors; (B) Two-photon fluorescent imaging of HepG2 cells after being pretreated with $100 \mu \mathrm{mol} / \mathrm{L} \mathrm{CoCl}_{2}$ for different time followed by co-incubated with Nap-F $(2 \mu \mathrm{mol} / \mathrm{L})$ for $30 \mathrm{~min}$; (C) Two-photon fluorescent imaging of HepG2 cells after being pretreated with different concentration of $\mathrm{CoCl}_{2}$ for $48 \mathrm{~h}$ followed by co-incubated with Nap-F (2 $\mu \mathrm{mol} / \mathrm{L}$ ) for $30 \mathrm{~min}$; (D) Average intensity in different incubation time found in Figure 4B, C; that of $t=0 \mathrm{~h}$ or $0 \mu \mathrm{mol} / \mathrm{L}$ average intensity was defined as 1.0. $\lambda_{\mathrm{ex}}: 820 \mathrm{~nm}, \lambda_{\mathrm{em}}: 450 \sim 600 \mathrm{~nm}$.

\section{4 肿瘤组织成像}

最后, 为了展示双光子荧光探针在深层组织成像的 优势, 我们将探针 Nap-F 应用于肿瘤组织成像. 在 $Z$ 扫 描模式下, 双光子苂光信号强度随扫描深度的变化如图 5 所示. 结果表明, 探针 Nap-F 能够很好的染色肿瘤组 织, 并且成功实现双光子苂光成像肿瘤组织中的弗林蛋 白酶活性, 成像深度范围为 $30 \sim 140 \mu \mathrm{m}$. 这一系列成像 结果不仅证明了探针 Nap-F 具有优异的双光子性质, 也表明了其具有深层组织成像的能力.

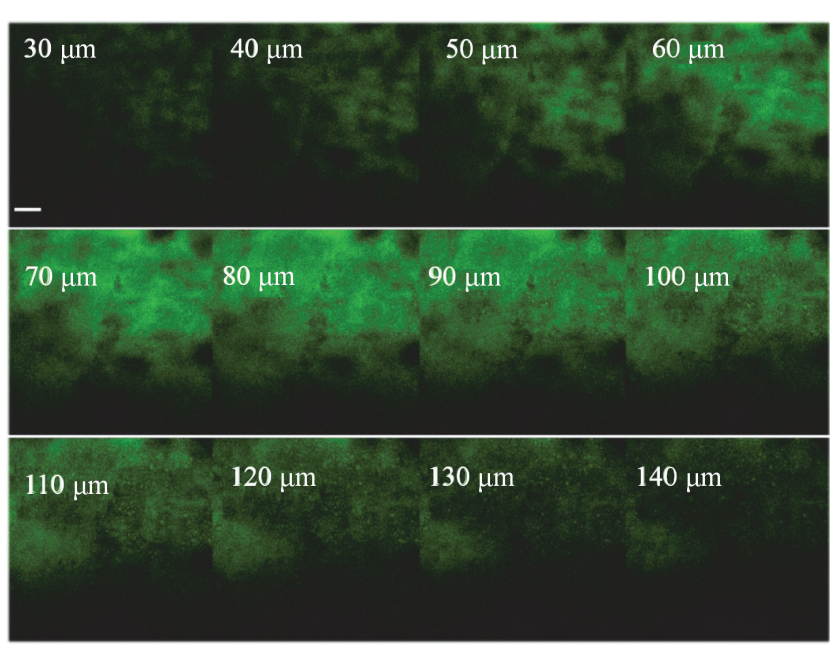

图 5 肿瘤组织 30 $140 \mu \mathrm{m}$ 范围的双光子苂光成像; 4T- 1 肿瘤组织与 探针 Nap-F $(20 \mu \mathrm{mol} / \mathrm{L})$ 在 $37{ }^{\circ} \mathrm{C}$ 下共孵育 $2 \mathrm{~h}$ 后成像, $\lambda_{\mathrm{ex}}: 820 \mathrm{~nm}, \lambda_{\mathrm{em}}$ : $450 \sim 600 \mathrm{~nm}$, 比例尺: $100 \mu \mathrm{m}$.

Figure 5 Two-photon fluorescence imaging of 4T-1 tumor tissues after co-incubated with Nap-F $(20 \mu \mathrm{mol} / \mathrm{L})$, the images showed the different depths of tumor tissue from 40 to $140 \mu \mathrm{mol} / \mathrm{L} . \lambda_{\mathrm{ex}}: 820 \mathrm{~nm}, \lambda_{\mathrm{em}}: 450 \sim 600$ $\mathrm{nm}$, scale bar: $100 \mu \mathrm{m}$.

\section{3 结论}

在本工作中, 我们构建了一个新型双光子荧光探针 Nap-F 用于细胞内弗林蛋白酶的检测与双光子成像. Nap-F 是由经典双光子苂光染料 1,8 -荎酰亚胺、弗林蛋 白酶特异性多肽序列 RVRR 和自消除连接体整合而成. 同时, 通过改变底物序列顺序 (RRRV), 我们还合成了 对比探针 Nap-C. 在缓冲溶液体系中, 我们证实了探针 Nap-F 对弗林蛋白酶具有高度的特异性, 能够定量检测 弗林蛋白酶的活性. 此外, 探针表现出很好的稳定性, 在不含目标物的检测体系中放置 $3 \mathrm{~d}$ 仍未见假阳性信号. 采用飞秒激光 $820 \mathrm{~nm}$ 激发下, Nap-F 能有效降低生物背 景, 并提高组织穿透深度, 适用于细胞和组织的双光子 成像. 探针成功地实现了对几种活细胞中弗林蛋白酶的 双光子成像, 揭示了癌细胞和表达缺陷细胞中弗林蛋白 酶含量的差异，表明弗林蛋白酶可作为监测肿瘤发生、 发展的靶标分子. 更重要的是, 我们将该探针用于 $\mathrm{CoCl}_{2}$ 固定 HIF-1 构建的肿瘤细胞缺氧模型成像, 实验 结果表明弗林蛋白酶的表达与肿瘤细胞缺氧程度存在 正相关性. Nap-F 能够解决基于硝基还原酶的缺氧探针 假阳性等问题，有望成为一种高保真的肿瘤缺氧探针. 双光子肿瘤组织成像结果表明 Nap-F 具有很好的组织穿 透深度. Nap-F 有望应用于生物医学样品中弗林蛋白酶 的检测, 成为监测肿瘤发生、转移和诊断的有力工具.

\section{4 实验部分}

\section{1 探针的合成}

探针的合成路线如支持信息图 S2 所示. 双光子苂 光染料 3 按照文献报道方法合成 ${ }^{[28]}$. ${ }^{1} \mathrm{H}$ NMR 
(DMSO-d6, $400 \mathrm{MHz}) \delta: 11.92(\mathrm{~s}, 1 \mathrm{H}), 8.55 \sim 8.51(\mathrm{~d}, J=$ $8.52 \mathrm{~Hz}, 1 \mathrm{H}), 8.48 \sim 8.44(\mathrm{~d}, J=8.45 \mathrm{~Hz}, 1 \mathrm{H}), 8.37 \sim 8.33$ $(\mathrm{d}, J=8.34 \mathrm{~Hz}, 1 \mathrm{H}), 7.79 \sim 7.73(\mathrm{t}, J=7.75 \mathrm{~Hz}, 1 \mathrm{H})$, $7.20 \sim 7.16(\mathrm{~d}, J=7.16 \mathrm{~Hz}, 1 \mathrm{H}), 4.06 \sim 4.02(\mathrm{t}, J=4.02$ $\mathrm{Hz}, 2 \mathrm{H}), 1.65 \sim 1.62(\mathrm{t}, J=1.60 \mathrm{~Hz}, 2 \mathrm{H}), 1.40 \sim 1.34(\mathrm{~m}$, $2 \mathrm{H}), 0.99 \sim 0.96(\mathrm{t}, J=0.94 \mathrm{~Hz}, 3 \mathrm{H}) ;{ }^{13} \mathrm{C} \mathrm{NMR}(400 \mathrm{MHz}$, DMSO-d6): $\delta$ : 164.07, 163.40, 160.66, 133.89, 131.46, $129.57,129.24,125.93,122.79,122.21,113.04,110.37$, 39.54, 30.21, 20.29, 14.16. MS (ESI) $\mathrm{m} / \mathrm{z}: 268.14$ (calcd for $\mathrm{C}_{16} \mathrm{H}_{15} \mathrm{NO}_{3}[\mathrm{M}-\mathrm{H}]^{-}$269.11).

Pbf 保护的 AcRVRR 序列和化合 4 均按照我们已报 道的方法 (多肽固相合成法) 合成 ${ }^{[12,13]}, \mathrm{Pbf}$ 保护的 AcRVRR 质谱结果为 MS (ESI) $m / z$ : 1383.58 (calcd for $\mathrm{C}_{64} \mathrm{H}_{97} \mathrm{~N}_{13} \mathrm{O}_{15} \mathrm{~S}_{3}[\mathrm{M}+\mathrm{H}]^{+}$1383.64). 化合 4 质谱结果为 MS (ESI) $m / z$ : 1480.54 (calcd. for $\mathrm{C}_{70} \mathrm{H}_{109} \mathrm{~N}_{15} \mathrm{O}_{14} \mathrm{~S}_{3}[\mathrm{M}+$ $\mathrm{H}]^{+}$1479.74)

将化合物 3 (105.3 $\mathrm{mg}, 0.39 \mathrm{mmol})$ 和三光气 (500.0 $\mathrm{mg}, 1.68 \mathrm{mmol}$ ) 加入 $250 \mathrm{~mL}$ 双颈圆底烧瓶中, 在氮气 保护下经注射器加入 $25 \mathrm{~mL}$ 干燥二氯甲烷. 将瓶子放在 冰浴中, 然后通过注射器加入 $0.5 \mathrm{~mL}$ DIPEA. 将反应液 在冰浴中反应 $1 \mathrm{~h}$, 然后在室温下继续搅拌 $12 \mathrm{~h}$. 然后减 压蒸发有机溶剂至干燥, 以氢氧化钠溶液作为尾气装置 吸收光气. 用注射器向反应瓶中加入溶于 $20 \mathrm{~mL}$ 干燥二 氯甲烷中的化合物 $4(500.0 \mathrm{mg}, 1.68 \mathrm{mmol})$ 和 DIPEA $(0.5 \mathrm{~mL})$, 室温摚拌 $10 \mathrm{~h}$ 后, 减压浓缩反应混合物, 得到 固体残渣. 将固体残留物重新溶解在二氯甲烷/三氟乙 酸 $(V$ 二氯甲烷 $: V$ 三皮乙酸 $=1: 99)$ 中的混合溶液中, 再加入 三异丙基硅烷 (TIPS, $100 \mu \mathrm{L}$ ), 室温下搅拌 $3 \mathrm{~h}$, 减压蒸 馏去掉溶剂, 通过高效液相色谱法 (HPLC) 提纯产物, 产量: $171.5 \mathrm{mg}(67 \%)$. 探针 Nap-F 的质谱结果为 $\mathrm{MS}$ (ESI) $m / z: 1019.53$ (calcd for $\mathrm{C}_{48} \mathrm{H}_{74} \mathrm{~N}_{16} \mathrm{O}_{9}[\mathrm{M}+\mathrm{H}]^{+}$ 1018.58). 对照探针 Nap-C 的合成方法与 Nap-F 一致, 仅换用序列为 RRRV 的多肽序列即可.

\section{2 缓冲溶液中的响应性能考察}

本实验中以 MDA-MB-468 细胞裂解提取液作为弗 林蛋白酶来源. 将 MDA-MB-468 细胞接种生长到 $90 \%$ 细胞密度时进行裂解实验. 将培养血中培养基吸出并弃 置, 用 DPBS 漂洗细胞三次并保证尽可能少的 DPBS 残 留液; 随后加入 $200 \mu \mathrm{L}$ RIPA 裂解液, 置于摇床低速均 匀摇晃 $10 \sim 15 \mathrm{~min}$, 使细胞充分裂解. 裂解结束后, 收 集裂解液, 并以 $13000 \mathrm{r} / \mathrm{min}$ 转速进行离心, 取上层澄清 悬浮液, 放置在 $-80{ }^{\circ} \mathrm{C}$ 保存. 实验中所需的其他细胞 裂解液与上述操作相同.

将探针 Nap-F 和 Nap-C 配制成 $1 \times 10^{-4} \mathrm{~mol} / \mathrm{L}$ 的 DMSO 储备液, $4{ }^{\circ} \mathrm{C}$ 下避光保存备用. 紫外光谱及苂光 光谱测定均在标准缓冲液 $(20 \mathrm{mmol} / \mathrm{L}$ Tris- $\mathrm{HCl}, \mathrm{pH}=$ 7.4, $1 \mathrm{mmol} / \mathrm{L} \mathrm{Ca}^{2+}, 5 \mathrm{mmol} / \mathrm{L} \mathrm{Mg}^{2+}, 0.1 \%$ Triton $\mathrm{X}-100$ ) 中进行, 反应温度为 $37{ }^{\circ} \mathrm{C}$. 激发波长设置为 $420 \mathrm{~nm}$,
发射波长接收范围设置为 $450 \sim 650 \mathrm{~nm}$, 狭缝宽度均为 $3 \mathrm{~nm}$. 选择性实验中用到的测试物用二次水溶解稀释成 所需浓度. 测量时, 探针最终浓度为 $5 \mu \mathrm{mol} / \mathrm{L}$.

\section{3 高效液相色谱 (HPLC)分析}

用体积分数为 $1 \% \mathrm{o}$ 的 TFA 水溶液和乙腈溶液作为实 验流动相; 实验所用色谱分离柱为反向分离柱. 对化合 物 3, Nap-F 以及 Nap-F 与细胞裂解的反应体系依次进行 分离和定性分析. 本实验采用梯度洗脱来实现各成分间 的分离; 通过调节流动相 $\mathrm{H}_{2} \mathrm{O} / \mathrm{CH}_{3} \mathrm{CN}$ 的体积比, 从 9 : 1 到 $4: 6$, 从而达到较好的分离效果. 利用紫外检测系 统收集样品中吸光度位于 $390 \mathrm{~nm}$ 处的实时信息.

\section{4 细胞毒性实验}

采用 MTS (3-(4,5-dimethylthiazol-2-yl)-5-(3-carboxymethoxyphenyl)-2-(4-sulfophenyl)-2H-tetrazolium)法 评估探针 Nap-F 的细胞毒性. 将 MDA-MB-468 细胞以 每孔 $1 \times 10^{4}$ 个细胞接种于 96 孔板, $24 \mathrm{~h}$ 后用不同浓度 $(2.5 \sim 20 \mu \mathrm{mol} / \mathrm{L})$ 的 Nap-F 共孵育 $24 \mathrm{~h}$. 随后, 弃去孔中 培养基, 并以 $200 \mu \mathrm{L}$ DPBS 洗涤小孔三次, 再向每孔中 加入 $20 \mu \mathrm{L}$ MTS 和 $80 \mu \mathrm{L}$ 培养基, 并在 $37^{\circ} \mathrm{C}$ 恒温培养 箱中孵育 $2 \mathrm{~h}$. 用酶标仪测量 $490 \mathrm{~nm}$ 处的吸光度, 进而 计算细胞的存活率.

\section{5 细胞成像实验}

将 HeLa、HepG2、Lovo 和 MDA-MB-468 四种细胞 分别接种至光学培养血中并培养至密度约为 $80 \%$. 用 DPBS 漂洗细胞, 换用新鲜培养基后，加入 Nap-F 于 $37{ }^{\circ} \mathrm{C}$ 恒温培养箱中与其共孵育 $30 \mathrm{~min}$, 用 DPBS 洗涤 三次, 然后于茨光共聚焦显微镜上进行细胞成像. 探针 的成像浓度为 $5 \mu \mathrm{mol} / \mathrm{L}$, 单、双光子苂光共聚焦成像激 发波长分别为设置为 405 和 $820 \mathrm{~nm}$, 发射接收波长分 别为 $500 \sim 600 \mathrm{~nm}$ 和 $450 \sim 600 \mathrm{~nm}$.

在 $\mathrm{CoCl}_{2}$ 构建的细胞缺氧模型成像实验中, 先将 $\mathrm{HepG}_{2}$ 细胞接种至光学培养血中培养至密度约为 $80 \%$, 然后用 DPBS 漂洗细胞, 为了诱导细胞内 HIF-1 不同程 度的固定化, 以不含 FBS 的新鲜 DMEM 培养基培养细 胞，与 $100 \mu \mathrm{mol} / \mathrm{L}$ 的 $\mathrm{CoCl}_{2}$ 共同孵育 $0,4,8,12,24$ 和 48 $\mathrm{h}$; 或与不同浓度的 $\mathrm{CoCl}_{2}(0,10,25,50,75$ 和 100 $\mu \mathrm{mol} / \mathrm{L}$ ) 共孵育 $48 \mathrm{~h}$, 然后更换培养基 $10 \%$ 并加入探 针 Nap-F (最终浓度为 $2 \mu \mathrm{mol} / \mathrm{L}$ ) 继续孵育 $30 \mathrm{~min}$, 再进 行细胞成像. 仪器参数与上述细胞成像参数一致.

\section{6 肿瘤组织成像实验}

将 $1 \times 10^{5}$ 个 $4 \mathrm{~T}-1$ 细胞分散在 $100 \mu \mathrm{L}$ 的 DPBS 中, 注射到五周大的雄性 BALB/c 鼠 (SJA Co., Ltd. Changsha, China) 左上肢. 4 周后, 肿瘤长到 $600 \mathrm{~mm}^{3}$ 大小, 然后剥出肿瘤, 冰冻 $24 \mathrm{~h}$, 制备切片. 待冷冻的肿瘤组 织恢复至室温后，与 $20 \mu \mathrm{mol} / \mathrm{L} \mathrm{Nap}-\mathrm{F}$ 在 $37{ }^{\circ} \mathrm{C}$ 下共孵育 $2 \mathrm{~h}$, 用 DPBS 漂洗切片 3 次, 随后于进行苂光成像, 用 Z-扫描模式收集不同组织深度处的苂光图像. 组织成像 
的激发波长与发射波长与上述细胞成像一致.

\section{References}

[1] Steiner, D. F. Curr. Opin. Chem. Biol. 1998, 2, 31

[2] Seidah, N. G.; Mayer, G.; Zaid, A.; Rousselet, E.; Nassoury, N.; Poirier, S.; Essalmani, R.; Prat, A. Int. J. Biochem. Cell Biol. 2008, 40, 1111.

[3] Bassi, D. E.; Fu, J.; de Cicco, R. L.; Klein-Szanto, A. J. P. Mol. Carcinogen 2005, 44, 151.

[4] McMahon, S.; Grondin, F.; McDonal, P. P.; Richard, D. E.; Dubois, C. M. J. Biol. Chem. 2005, $280,6561$.

[5] Zhang, J.; Liu, H.-W.; Hu, X.-X.; Li, J.; Liang, L.-H.; Zhang, X.-B.; Tan, W. Anal. Chem. 2015, 87, 11832.

[6] Li, X.; Gao, X.; Shi, W.; Ma, H. Chem. Rev. 2014, 114, 590.

[7] Yang, L.; Liu, B.; Li, N.; Tang, B. Acta Chim. Sinica, 2017, 75, 1047. (in Chinese). (杨立敏, 刘波, 李娜, 唐波. 化学学报, 2017, $75,1047$.

[8] Yang, Z.; He, Y.; Dai, B.; Dou, B.; Wang, J.; Peng, X. Acta Chim. Sinica, 2011, 69, 445 (in Chinese). (杨志刚, 和艳霞, 戴涁, 窦柏 荵, 王静云, 彭孝军. 化学学报, 2011, 69, 445.)

[9] Guan, X.; Wang, L.; Li, Z.; Liu, M.; Wang, K.; Lin, B.; Yang, X.; Lai, S.; Lei, Z.. Acta Chim. Sinica, 2019, 77, 1036 (in Chinese). (关 晓琳, 王林, 李志飞, 刘美娜, 王凯龙, 林斌, 杨学琴, 来守军, 雷自强. 化学学报, 2019, 77, 1036.)

[10] Hou, J.; Li, K.; Qin, C. Yu, X.; Chin. J. Org. Chem. 2018, 38, 612 (in Chinese). (后际挺，李坤，覃彩芹，余孝其. 有机化学, 2018, $38,612$.

[11] Liu, H.-W.; Chen, L.; Xu, C.; Li, Z.; Zhang, H.; Zhang, X.-B.; Tan, W. Chem. Soc. Rev. 2018, 47, 7140.

[12] Zhu, L.; Liu, H.-W.; Yang, Y.; Hu, X.-X.; Li, K.; Xu, S.; Li, J.-B.; Ke, G.; Zhang, X.-B. Anal. Chem. 2019, 91, 9682.
[13] Li, K.; Hu, X.-X.; Liu, H.-W.; Xu, S.; Huan, S.-Y.; Li, J.-B.; Deng, T.-G.; Zhang, X.-B. Anal. Chem. 2018, 90, 11680.

[14] Zhao, X.; Lv, G.; Peng, Y.; Liu, Q.; Li, X.; Wang, S.; Li, K.; Qiu, L.; Lin, J. ChemBioChem 2018, 19, 1060.

[15] Mu, J.; Liu, F.; Rajab, M. S.; Shi, M.; Li, S.; Goh, C.; Lu, L.; Xu, Q.-H.; Liu, B.; Ng, L. G., Xing B. Angew. Chem. Int. Ed. 2014, 53, 14357.

[16] Liu, X.; Liang, G. Chem. Commun. 2017, 53, 1037.

[17] Liu, H.-W.; Liu, Y.; Wang, P.; Zhang, X.-B. Methods Appl. Fluoresc. 2017, 5, 012003.

[18] Kim, H.; Cho, B. Chem. Rev. 2015, 115, 5014.

[19] Kim, H.; Cho, B. Acc. Chem. Res. 2009, 42, 863.

[20] Liu, H.-W.; Zhang, X.-B.; Zhang, J.; Wang, Q.-Q.; Hu, X.-X.; Wang, P.; Tan, W. Anal. Chem. 2015, 87, 8896.

[21] Huang, C.; Chen, H.; Li, F. An, S. Chin. J. Org. Chem. 2019, 39, 2467 (in Chinese). (黄池宝, 陈会, 李福琴, 安思雅. 有机化学, 2019, 39, 2467.)

[22] Dragulescu-Andrasi, A.; Kothapalli, S.-R.; Tikhomirov, G. A.; Rao, J.; Gambhir, S. S. J. Am. Chem. Soc. 2013, 135, 11015.

[23] Yuan, Y.; Zhang, J.; Cao, Q.; An, L.; Liang, G. Anal. Chem. 2015, 87,6180 .

[24] Xu, S.; Liu, H.-W.; Hu, X.-X.; Huan, S.-Y.; Zhang, J.; Liu, Y.-C.; Yuan, L.; Qu, F.-L.; Zhang, X.-B.; Tan, W. Anal. Chem. 2017, 89, 7641

[25] Thorn-Seshold, O.; Vargas-Sanchez, M.; McKeon, S.; Hasserodt, J. Chem. Commun. 2012, 48, 6253.

[26] Ou-Yang, J.; Li, Y.-F.; Wu, P.; Jiang, W.-L.; Liu, H.-W.; Li, C.-Y. ACS Sens. 2018, 3, 1354 .

[27] Ma, J.; Evrard, S.; Badiola, I.; Siegfried, G.; Khatib, A. M. Eur. J. Cell Biol., 2017, 96, 457.

[28] Feng, L.; Li, P.; Hou, J.; Cui, Y.-L.; Tian, X.-G.; Yu, Z.-L.; Cui, J.-N.; Wang, C.; Huo, X.-K.; Ning, J., Ma X.-C. Anal. Chem. 2018, $90,13341$.

(Yang, X.) 